\title{
Expression Profiles of Craniosynostosis-Derived Fibroblasts
}

\author{
Francesco Carinci, ${ }^{1}$ Maria Bodo, ${ }^{2}$ Lara Tosi, ${ }^{3,4}$ Francesca Francioso, ${ }^{4}$ Rita Evangelisti, ${ }^{5}$ \\ Furio Pezzetti, ${ }^{3,4}$ Luca Scapoli, $^{3,5}$ Marcella Martinelli, ${ }^{5}$ Tiziano Baroni, $^{2}$ Giordano Stabellini, ${ }^{6}$ \\ Paolo Carinci, ${ }^{3,4}$ Catia Bellucci, ${ }^{2}$ Cinzia Lilli, ${ }^{2}$ and Stefano Volinia ${ }^{5,7}$ \\ ${ }^{1}$ Chair of Maxillofacial Surgery, University of Ferrara, Italy \\ ${ }^{2}$ Institute of Histology and General Embryology, University of Perugia, Italy \\ ${ }^{3}$ Centre of Molecular Genetics, CARISBO Foundation, Bologna, Italy \\ ${ }^{4}$ Institute of Histology and General Embryology, University of Bologna, Italy \\ ${ }^{5}$ Functional Genomics, Section of Histology, Department of Morphology and Embryology, \\ University of Ferrara, Italy \\ ${ }^{6}$ Department of Anatomy, University of Milan, Italy \\ ${ }^{7}$ TIGEM, Telethon Institute of Genetics and Medicine, Napoli, Italy
}

Accepted August 26, 2002

\begin{abstract}
Background: Craniosynostosis syndromes, a group of connective disorders characterized by abnormalities in vault osteogenesis and premature fusion of bone sutures, are associated with point mutations in FGF receptor family members. The cellular phenotype is characterized by abnormal extracellular matrix turnover.

Material and Methods: We used primary cultures of periosteal fibroblasts derived from two different craniosynostosis syndromes, the Apert and Crouzon syndromes. The FGFR2 third immunoglobulin-like domain and its flanking linker regions were analyzed for mutation. DNA microarrays containing 19,200 cDNAs were used to study the gene expression profiles of Apert and Crouzon fibroblasts. The pathologic cells were compared to wild-type human periosteal fibroblasts.

Results: The P253R missense mutation and the G338R mutation were observed in Apert and Crouzon fibroblasts,
\end{abstract}

respectively. The genetic profiles, as evaluated by DNA microarrays, yielded different clusters of expressed sequence tag (ESTs) expression within the experiment. Expression profiles from craniosynostosis-derived fibroblasts differ from those of wild-type fibroblasts $(288 \mathrm{hu}-$ man ESTs, $p<0.01$, pFDR $=0.12$ ). Furthermore, two ESTs clusters discriminate the Crouzon from Apert fibroblasts. The differentially expressed genes cover a broad range of functional activities, including (1) bone differentiation, (2) cell-cycle regulation, (3) apoptotic stimulation, and (4) signaling transduction, cytoskeleton, and vesicular transport.

Conclusions: The transcriptional program of craniosynostosis fibroblasts differs from that of wild-type fibroblasts. Expression profiles of Crouzon and Apert fibroblasts can also be distinguished by two EST expression clusters, thus hinting at a different genetic background.

\section{Introduction}

Mechanisms involving growth factors and different time-space expression of extracellular matrix (ECM) components participate in skull osteogenesis. Cranial sutures contain osteogenic stem cells and periosteal fibroblasts that, during human embryo development, differentiate into osteoblasts that produce premineralized osseous matrix. Abnormal ossification leads to premature fusion of cranial sutures and craniosynostosis syndromes such as the Apert and Crouzon syndromes.

The Apert syndrome is characterized by acrobrachycephalic vault, exorbitism, hypertelorism, and dystopy of the outer canthus; the nose is hooked and the mouth has the shape of an isosceles triangle.

Correspondence and reprint requests should be addressed to: Maria Bodo, Sezione di Istologia ed Embriologia Sperimentale, Via del Giochetto 06100 Perugia, Italy. Phone: (39)075-585.7432; fax: (39)075-585.7434; e-mail: bodo@unipg.it.
Crouzon patients have brachycephaly, ocular proptosis, teleorbitism, and midfacial hypoplasia. The Apert clinical phenotype is more severe than the Crouzon, not only at the cranial level, but also in the limbs where there is syndactyly of hands and feet. Cranial base abnormalities are more severe and synchondroses of cranial base are calcified at an earlier age, before vault sutures (1). In the Crouzon syndrome coronal and lambdoid sutures are the most affected (2).

ECM molecule composition plays a role in the morphogenetic processes that occur during skull development. Differences in ECM synthesis and secretion of glycosaminoglycans, collagens, proteoglycans, and fibronectin in fibroblasts and osteoblasts from Apert and Crouzon patients have been reported (3-7). These changes in ECM were also affected by exposure to growth factors such as transforming growth factor beta (TGF- $\beta)(8)$, interleukins $(3,4,5,7)$, 
and the basic fibroblast growth factor (bFGF) $(6,9)$. bFGF, the most abundant growth factor in the vault, regulates cell proliferation, migration, and differentiation; overall it has diverse roles in regulating bone osteogenesis (10). As growth factors are secreted in an autocrine-paracrine manner and interact with ECM, the ECM pattern may be considered another signal of morphogenetic control. Indeed, it is a further variable in phenotype determination.

The molecular basis for craniosynostosis has been partially elucidated. Many human craniosynostosis disorders have been linked to activating mutations in FGF receptors (FGFR) 1 and 2, but the precise effects of FGF on the proliferation, maturation, and differentiation of the target osteoblastic cells are still under investigation (11). Interestingly, defects in the same gene induce craniosynostosis syndromes with a wide spectrum of clinical phenotypes and indeed several different point mutations have been mapped in FGFRs involved in cranial development disorders. FGFRs are composed of a single transmembrane domain, an extracellular domain composed of three immunoglobulin (Ig)-like domains, and an intracellular domain containing two tyrosine kinase subdomains. In the Crouzon syndrome, several mutations in the third Ig-like domain of the extracellular portion of FGFR2 have been identified $(12,13)$. In patients with Apert syndromes, FGFR2 mutations produce missense substitutions involving adjacent amino acids in the linker region between the second and third extracellular Ig domain (14). Moreover, genes other than FGFR 1-4, such as MSX2 and TWIST, can also cause craniosynostosis (15).

In this study we analyzed the expression profiles of periosteal fibroblasts from patients with craniosynostosis (Apert and Crouzon syndromes) and compared them to those of wild-type periosteal fibroblasts. Furthermore, we aimed to identify FGFRregulated transcripts for investigating the molecular mechanism underlying abnormal FGFR signaling.

\section{Materials and Methods Cell Culture}

Human periosteal fibroblasts were obtained from the galea-pericranium of cell parietal bones of two Crouzon patients (Crouzon Q and Crouzon K) and one Apert patient (all aged 17-18 years) during corrective surgery. Wild-type control fibroblasts originated from explants of periosteum removed from two age-matched subjects at the time of surgery for repair of parietal bone fractures. Primary cultures were grown in Falcon flasks (Becton Dickinson, Franklin Lakes, NJ, USA) containing minimum essential medium (MEM) supplemented with $20 \%$ fetal calf serum (Gibco, Paisley, UK), essential amino acids, vitamins, and antibiotics (Serva, Heidelberg, Germany). Subcultures were obtained 20-30 days later. All tests were performed between the sixth and eighth subculture. All fibroblasts were then incubated with MEM with $0.5 \%$ fetal calf serum for $24 \mathrm{hr}$.

\section{Mutation Detection}

Genomic DNA was extracted from Crouzon and Apert periosteal fibroblasts according to standard procedures (16). The FGFR2 third Ig-like domain and its flanking linker regions were analyzed for mutations. Primers designed from intronic sequences flanking exon IIIa (forward 5' -acccagttgtgggtacctt-3'; reverse $5^{\prime}$-tccccactcctccttcttc $3^{\prime}$ ) and exon IIIc (forward 5'-aaaaggggccatttctgataa-3'; reverse 5'-ccacaatcattcctgtgtcg-3) were used to amplify genomic DNA by PCR. Direct sequencing of the purified PCR product was performed on both forward and reverse strand on an ABI377 sequencer using the Big Die Terminator sequencing Kit.

\section{DNA Microarrays}

Expression profiling was performed essentially as previously described (17). Briefly, total RNA was extracted from normal and pathologic cells cultured as above, using RNAzol. Ten micrograms of total RNA were used for each sample. cDNA was synthesized by using Superscript II (Invitrogen, Paisley, England, UK) and amino-allyl dUTP (Sigma, St. Louis, MO, USA). Monoreactive Cy 3 and Cy 5 esters (Amersham Biosciences, Little Chalfont, England, UK) were used for indirect cDNA labeling. RNA from wild-type fibroblasts was labeled with $\mathrm{Cy} 3$ and used as common reference against every other Cy5-labeled cDNAs. Human 19.2K DNA microarrays, containing 19,200 expressed sequence tag (ESTs), corresponding to at least 15,448 different Unigene clusters, were used (Ontario Cancer Institute, Ontario, Canada). One hundred microliters of the sample and control cDNA in DIG Easy hybridization solution (Roche, Basel, Switzerland) were used at $37^{\circ} \mathrm{C}$ overnight. Washing was performed three times for 10 min with $1 \times$ SSC, $0.1 \%$ SDS at $42{ }^{\circ} \mathrm{C}$, and three times for 5 min with $0.1 \times$ SSC at room temperature. Hybridized slides were scanned using GenePix 4000A (Axon Instruments, Foster City, CA, USA). Arabidopsis RNA was used as a reference for RNA labeling.

Images were analyzed by GENEPIX PRO 3.0 (Axon Instruments) and data extracted as previously described (17). Four thousand four hundred and eight arrayed ESTs were present in at least $75 \%$ of the samples. Spotted ESTs with expression levels higher than reference have a positive $\log 2 \mathrm{Cy} 5 / \mathrm{Cy} 3$ ratio, whereas those with lower expression have a negative $\log 2 \mathrm{Cy} 5 / \mathrm{Cy} 3$ ratio.

To select the ESTs that distinguish between the craniosynostosis and the wild-type fibroblasts, and the EST that distinguish Crouzon and Apert phenotypes, we used $t$-test combined with bootstrap analysis for assigning $p$-values. Multiple $t$-testing correction (18) was also applied. Positive pFDR was defined as the proportion of ESTs expected to be identified by chance (assuming probes are independent) relative to 
the total number of probes called significant. A balanced bootstrap analysis with 50,000 permutations was applied to the tests to define the number of expected false positives by chance. Expression tables were then analyzed using hierarchical clustering and principal component analysis (PCA).

\section{Results}

\section{Mutation Detection}

Fibroblasts from the Apert patient showed a C-to-G transversion that causes the P253R missense substitution in the linker between the second and third extracellular immunoglobulin domains of FGFR2. A C-to-G transversion was observed in fibroblasts from one Crouzon patient (patient Q) resulting in the missense mutation G338R. No mutation was identified in fibroblasts from the second Crouzon patient (patient K). The two Crouzon patients were shown to be unrelated by DNA polymorphism analysis (data not shown).

\section{cDNA Microarrays}

We identified differences in EST expression between craniosynostosis and wild-type fibroblasts by using $t$-test analysis. To perform the analysis, three craniosynostosis samples were compared to two wild-type samples. Two hundred eighty-eight ESTs were differentially expressed in craniosynostosis fibroblast cultures $(p<0.01$; pFDR $=0.12)$ out of 4408 measured ESTs. Figure l shows a hierarchical tree representation of the divergent EST clusters in Crouzon Q and K, and Apert fibroblasts when compared to wild-type fibroblasts.

The genetic program modulation in craniosynostosis cells, which results in two clusters of up- and down-regulated ESTs, is illustrated in Figure 2 by the use of principal component analysis. ESTs that significantly differentiate between wild-type and craniosynostosis derived cells are listed in Table 1.

We then proceeded to identify possible divergences within craniosynostosis groups, in particular between Crouzon and Apert fibroblasts. A number of ESTs were identified with such pattern of expression, namely 59 ESTs were up-regulated and 21 down-regulated in Crouzon primary cultures when compared to Apert or wild-type fibroblasts $(p<0.01)$. Table 2 shows such a list of Crouzon-specific ESTs that are not regulated in either Apert or in the wild-type samples. Complete expression tables and results from statistical analysis are accessible at URL: http://biotecnologie.unife.it/microarrays/crouzon.

\section{Discussion}

The regulatory processes that underlie ossification and fusion of sutures during cranial vault morphogenesis are still largely obscure, but they are known to depend on microenvironment ECM. FGFRs have a role in controlling premature maturation at the growth plates of long bones and at the suture of the skull (19). Genetic data support the hypothesis that bFGF receptor mutation is involved in the pathophysiology of craniosynostosis (12-14).

We identified the P253R missense mutation in Apert fibroblasts and the G338R missense mutation in fibroblasts from one of the two Crouzon patients. The G338R mutation had previously been observed

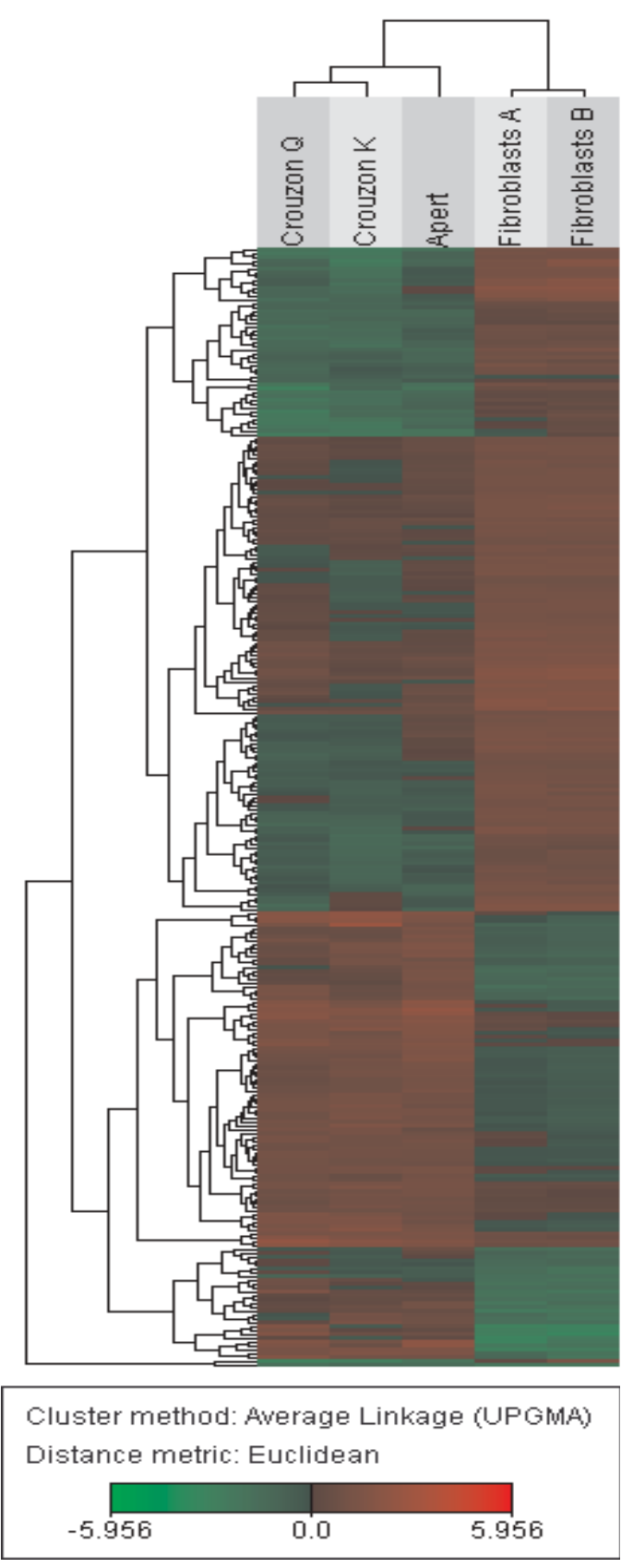

Fig. 1. Clustering of 288 spotted ESTs selected by $t$-test from 4408 ESTs in the expression table that were differentially expressed in craniosynostosis cells $(p<0.01$, average t-test significant spots in bootstrap, 37; pFDR, 0.12). Rows, ESTs; columns, individual patient samples; red, mRNA overexpression; green, underexpression. Crouzon Q, Crouzon K, and Apert indicate craniosynostosis derived fibroblasts. A and B fibroblasts indicate wild-type fibroblasts. 


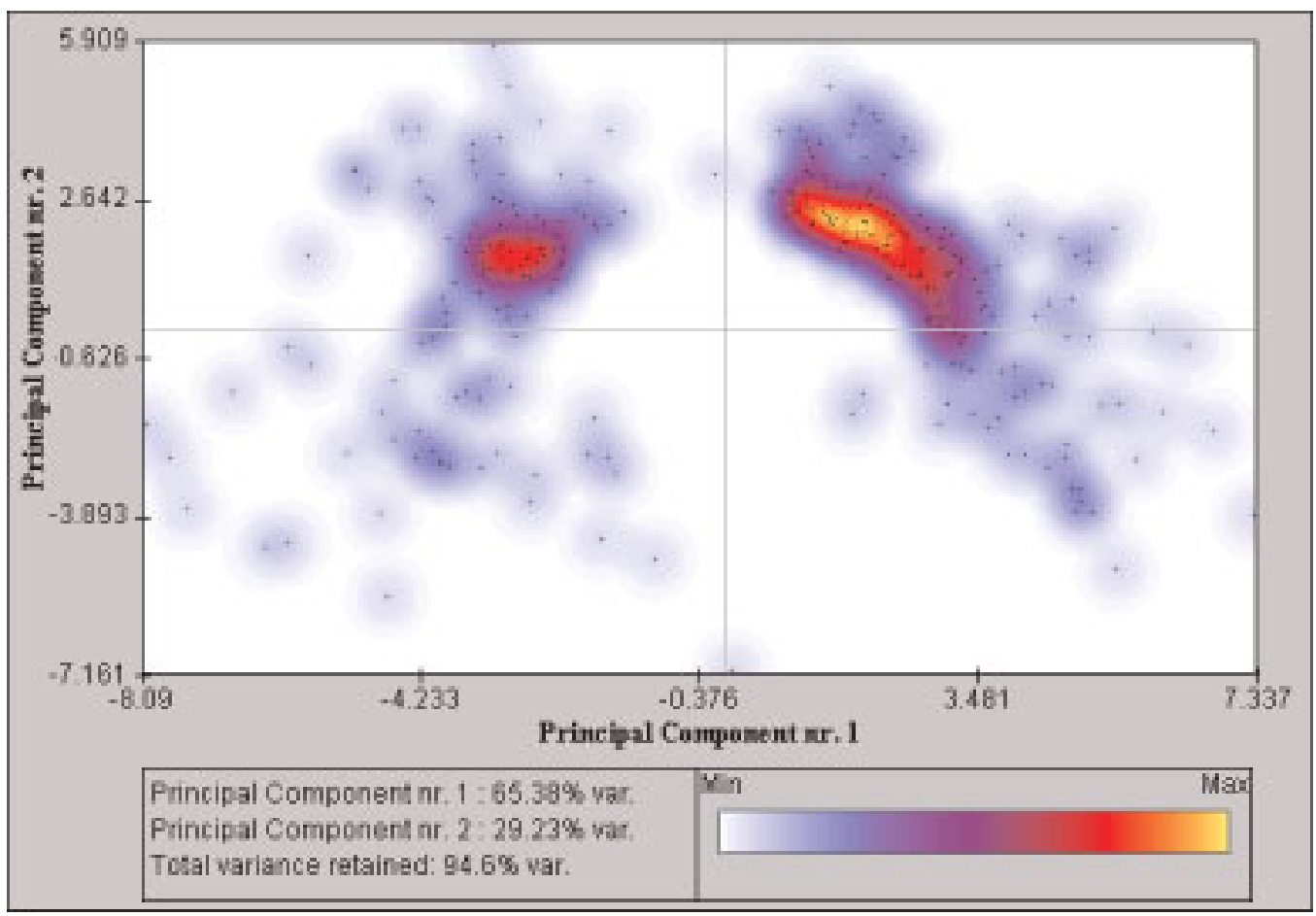

Fig. 2. Principal component analysis of differentially expressed ESTs in craniosynostosis primary fibroblasts. On the lefthand side of the graph, the group corresponding to 115 ESTs up-regulated in craniosynostosis and on the right-hand side that of the 173 down-regulated ESTs $(p<0.01)$.

in other patients with Crouzon syndrome (20). No mutation was found in fibroblasts from the second Crouzon patient analyzed by cDNA microarrays (patient $\mathrm{K})$. This is not surprising given the high allelic heterogeneity in the Crouzon syndrome (15), but presumably this patient presented a mutation in the uninvestigated part of the FGFR. Indeed, only about half of the mutations responsible for the Crouzon syndrome occur in FGFR2 exons IIIa and IIIc, which we examined.

Hybridization of mRNA-derived target to DNA microarrays allows systemic analysis of expression profiles for thousands of genes simultaneously and provides primary information on transcriptional changes. Several studies have already demonstrated the validity of this technique in identifying cancerrelated genes and in classifying human cancer at the molecular level $(17,21-24)$.

The present report presents a modern genetic approach to the study of skeletal dysplasias and helps to elucidate the normal mechanism of skeletal growth and development. We identified several genes whose expression, when compared with wild-type cells, was definitely deregulated in craniosynostosis-derived cells. These differentially expressed genes, cover a broad range of activities: (1) bone differentiation, (2) cell-cycle regulation, (3) apoptotic stimulation, (4) signaling transduction, (5) transcription, (6) translation, (7) metabolic change, and (8) cytoskeleton and vesicular transport.
Two of the up-regulated genes, the osteoclast stimulating factor 1 (OSTF1) and the growth differentiation factor 11 (GDF11) in craniosynostosisderived fibroblasts, are related to bone morphogenesis. OSTF1 is an intracellular protein that enhances 1,25 dihydroxyvitamin $\mathrm{D}_{3}$, induces bone resorption, binds c-Src with high affinity, and indirectly stimulates osteoclast formation (25). GDF11, a member of the bone morphogenetic protein family and the TGF- $\beta$ superfamily, is a secreted signal that acts to specify positional identity along the anterior/posterior axis (26).

Gene sets for signal transduction include both up- and down-regulated genes in craniosynostosis cells. Phosphatidylinositol 3-kinase is a lipid kinase that phosphorylates the inositol ring of phosphatidylinositol and related compounds at the $3^{\prime}$ position. The products of these reactions serve as second messengers in growth signaling pathways. The kinase itself is made up of a catalytic subunit of molecular weight $110 \mathrm{kD}$ and a regulatory subunit of either $85 \mathrm{kD}, 55 \mathrm{kD}$, or $50 \mathrm{kD}(27)$. The $85-\mathrm{kD}$ regulatory subunit of phosphatidylinositol 3-kinase is overexpressed in craniosynostosis cells.

On the other hand, calmodulin 2 is expressed at a lower than normal level in craniosynostosis cells. Calmodulin is the archetype of the family of calcium-modulated proteins. Nearly 20 members have been detected in the cytosol or on membranes facing the cytosol and identified by their high affinity 
Table 1. Differentially expressed genes in craniosynostosis versus wild-type cells: ESTs up-regulated in craniosynostosis cells (down in wild-type cells)

\begin{tabular}{|c|c|c|}
\hline Name & Symbol & GOabr \\
\hline $\begin{array}{l}\text { 3-Hydroxy-3-methylglutaryl-coenzyme A } \\
\text { synthase } 1 \text { (soluble) }\end{array}$ & HMGCS 1 & $\begin{array}{l}\text { Cytoplasm|soluble fraction|lipid metabolism| } \\
\text { hydroxymethylglutaryl-CoA synthase }\end{array}$ \\
\hline AD024 protein & $\mathrm{AD} 024$ & \\
\hline Aldehyde dehydrogenase 3 family, member Bl & ALDH3B 1 & $\begin{array}{l}\text { Lipid metabolism|alcohol metabolism|aldehyde } \\
\text { dehydrogenase }\end{array}$ \\
\hline $\begin{array}{l}\text { Amiloride binding protein } 1 \text { (amine oxidase } \\
\text { [copper-containing]) }\end{array}$ & $\mathrm{ABP} 1$ & Metabolism|peroxisome|amine oxidase|drug binding \\
\hline $\begin{array}{l}\text { ATP synthase, } \mathrm{H}^{+} \text {transporting, mitochondrial } \\
\text { F0 complex, subunit } \mathrm{f} \text {, isoform } 2\end{array}$ & ATP5J2 & \\
\hline Breast carcinoma amplified sequence 2 & BCAS2 & $\begin{array}{l}\text { Spliceosome|RNA processing|pre-mRNA splicing } \\
\text { factor }\end{array}$ \\
\hline $\begin{array}{l}\text { CDC } 37 \text { cell division cycle } 37 \text { homolog } \\
\text { (S. cerevisiae) }\end{array}$ & CDC 37 & $\begin{array}{l}\text { Chaperone|protein binding|protein targeting| } \\
\text { cell-cycle regulator|regulation of CDK activity }\end{array}$ \\
\hline Cell death-inducing DFFA-like effector b & CIDEB & Induction of apoptosis by DNA damage \\
\hline CGI-127 protein & LOC5 1646 & \\
\hline Chromosome 21 open reading frame 80 & $\mathrm{C} 21$ or $\mathrm{f} 80$ & \\
\hline Crystallin, alpha B & CRYAB & $\begin{array}{l}\text { Vision|nucleus|cytoplasm|chaperone|protein folding| } \\
\text { muscle contraction }\end{array}$ \\
\hline Cyclin D-type binding-protein 1 & CCNDBP1 & \\
\hline Cytochrome c oxidase subunit VIc & $\mathrm{COX} 6 \mathrm{C}$ & Energy pathways \\
\hline Dipeptidylpeptidase VI & DPP6 & Dipeptidyl-peptidase \\
\hline
\end{tabular}

After $t$-test selection, as explained in Results and Discussion, the gene graphs were individually confirmed by using JExpress. In this table, a selection of genes with the most highly significant $t$-test values is reported. The complete tables can be found at URL: http://nbiol29.unife.it/crouzon.

for calcium. Calmodulin plays a role in growth and the cell cycle as well as in signal transduction and the synthesis and release of neurotransmitters (28).

Our results showed that many translation genes are down-regulated in craniosynostosis fibroblasts, including genes coding for ribosomal proteins. The poly(A)-binding protein was also down-regulated. This protein is found complexed to the 3' poly(A) tail of eukaryotic mRNA which is required for poly $(\mathrm{A})$ shortening and translation initiation (29).

Among the down-regulated genes in craniosynostosis-derived cells, eukaryotic translation initiation factors that initiate protein synthesis and may increase specificity of target gene activation by AP-1 are also present (30). The transcription termination factor, RNA polymerase II (TTF2), which is one component of negative transcription elongation factor (N-TEF), is also down-regulated in craniosynostosis cells. Transcription elongation is an important target for control of eukaryotic gene expression. After initiating from a promoter, the RNA polymerase II elongation complex is subjected to a block of elongation by the action of components of N-TEF . One component of N-TEF is TTF2 (31).
Interestingly, cytoskeleton genes and genes involved in vesicular transport are down-regulated in craniosynostosis-derived cells. Cofilin is an actinmodulating protein that binds and depolymerizes filamentous F-actin and inhibits the polymerization of monomeric G-actin in a pH-dependent manner. It is involved in the translocation of actin-cofilin complex from cytoplasm to the nucleus (32). Profilin 1 , a ubiquitous actin monomer-binding protein belonging to the profilin family, is thought to regulate actin polymerization in response to extracellular signals. Dissociation of the profilin-actin complex is caused by binding of phosphatidylinositol 4,5-bisphosphate to profilin (33).

Overall, these results indicate that the genes involved in inducing osteoblast differentiation are upregulated in craniosynostosis-derived cells and confirm that the ECM turnover is abnormal (34). In fact, we reported an increase in collagen accumulation, altered synthesis of glycosaminoglycans and proteoglycans, and fibronectin production in craniosynostosis cells $(6,7)$. Furthermore, even the pattern of signal transduction phosphoprotein gene expression confirms our previous observations that the P85 beta isoform is expressed in Crouzon fibroblasts and is up-regulated by 
Table 2. Differentially expressed genes in Crouzon versus Apert fibroblasts: ESTs up-regulated in Crouzon fibroblasts

\begin{tabular}{|c|c|c|c|}
\hline Clone ID & Name & Cytoband & GOabr \\
\hline 41653 & Fatty acid desaturase 1 & $11 q 12.2-q 13.1$ & $\begin{array}{l}\text { C-5 sterol desaturase|fatty acid } \\
\text { desaturation|integral membrane protein }\end{array}$ \\
\hline 28193 & Ubiquitin-specific protease 28 & $11 q 23$ & \\
\hline 502965 & KIAA1391 protein & $11 q 23.2$ & \\
\hline 279904 & Enhancer of invasion 10 & $14 q 11.1$ & \\
\hline 154147 & $\begin{array}{l}\text { Golgi-associated, gamma adaptin ear- } \\
\text { containing, ARF binding protein } 2\end{array}$ & $16 \mathrm{p} 12$ & \\
\hline 469423 & LIS 1-interacting protein NUDE1, rat & $16 \mathrm{p} 13.11$ & \\
\hline 114341 & KIAA1321 protein & $17 q 11.1$ & \\
\hline 40462 & Zinc finger protein 264 & $19 q 13.4$ & \\
\hline 171569 & Janus kinase 1 (a protein tyrosine kinase) & $1 \mathrm{p} 32.3-\mathrm{p} 31.3$ & $\begin{array}{l}\text { Protein phosphorylation|protein } \\
\text { tyrosine kinase|intracellular signaling } \\
\text { cascade|peripheral plasma membrane } \\
\text { protein }\end{array}$ \\
\hline 429621 & Hypothetical protein FLJ23231 & $1 p 35.2$ & \\
\hline 178037 & RNA, U17D small nucleolar & $1 p 36.1$ & \\
\hline 194512 & PC326 protein & $1 \mathrm{q} 23.2$ & \\
\hline 172447 & Chromosome 20 open reading frame 154 & $20 \mathrm{p} 12.3$ & \\
\hline 203939 & RNA-binding region (RNPI, RRM) containing 2 & $20 q 11.21$ & $\begin{array}{l}\text { Nucleoplasm|RNA processing| } \\
\text { pre-mRNA splicing factor }\end{array}$ \\
\hline 152378 & Hypothetical protein FLJ20635 & $22 q 13$ & \\
\hline 381382 & Hypothetical protein & $22 \mathrm{q} 13.2$ & \\
\hline 112048 & Prenylcysteine lyase & $2 \mathrm{p} 13.3$ & \\
\hline 117322 & Eukaryotic translation initiation factor $4 \mathrm{E}$-like 3 & $2 q 36.1$ & $\begin{array}{l}\text { mRNA cap binding|translation factor| } \\
\text { translational regulation }\end{array}$ \\
\hline 156984 & Transketolase (Wernicke-Korsakoff) & $3 p 14.3$ & Transketolase \\
\hline 124459 & Chemokine binding protein 2 & $3 p 21.3$ & $\begin{array}{l}\text { Chemotaxis|immune response|plasma } \\
\text { membrane|chemokine receptor|devel- } \\
\text { opmental processes|integral plasma } \\
\text { membrane protein|G-protein linked } \\
\text { receptor protein signaling pathway }\end{array}$ \\
\hline 143261 & KIAA1 160 protein & $3 q 22.1$ & \\
\hline
\end{tabular}

Cluster of ESTs corresponding to the Crouzon Fibroblasts. These ESTs are significantly modulated $(p<0.01)$ in Crouzon fibroblasts when compared to Apert and wild-type fibroblasts. Fifty-nine ESTs and 21 ESTs are up-regulated in Crouzon fibroblasts. The IMAGE clone ID, attributes, cytoband, and gene ontology annotation where available are shown in the enclosed table.

bFGF treatment, as we found in studies analyzing the signaling pathways in mutant cells (9).

Finally, the present data confirm the cytoskeleton is a target and a mediator of the transduction mechanisms triggered by FGF signals. A cytoskeleton involvement in the distribution of cellular and extracellular ECM has been previously demonstrated (35) and this study indicates it has a role in the regulation of the craniosynostosis phenotype.

The different expression profiles in craniosynostosis cells and wild-type cells supports the hypothesis that craniosynostosis cells present either a different degree of constitutive activation or lower levels of mutated FGFR with a negative feed-back loop propagating downstream effectors. In other words, the abnormal receptor conformation could mimic and thus accentuate the effects of bFGF-FGFR binding or, on the contrary, reduce the levels of binding. In both scenarios, bone differentiation would be abnormal.

The intriguing difference between the two Crouzon and the Apert patients reported here for the first time suggests that the syndromes might be linked to different genetic backgrounds and might explain how identical FGFR mutations are associated with different clinical features. Many questions remain open. 
The consistent differences between Crouzon and Apert phenotypes are probably due to different molecular mechanisms of FGFR and to different downstream molecules in the FGFR signalling pathways. Studies on more patients which, given the rarity of craniosynostosis cannot be done in the short term, will provide data showing differences in the several groups with an extremely heterogeneous FGFR genotype more clearly.

Overall, the results of the present study indicate links between the molecular biology of the cellular phenotype and the mutated craniosynostosis genotype. Understanding how receptor mutations lead to the activation of specific genes involved in bone differentiation may provide important clues about the pathogenesis of bone disorders.

\section{Acknowledgments}

This study was supported, in part, by grants from TELETHON n. E.1147 (to P.C.), Fondazione CARISBO (to P.C.), Fondazione Cassa di Risparmio di Perugia (to M.B. SA 172901 project), MIUR ex $40 \% 2000$ (to P.C.), MIUR ex 60\% 2000 (to F.C.), and MIUR ex 60\%, PRIN 2000-2001 (to S.V.). We thank Dr Geraldine Boyd for the English revision.

\section{References}

1. Avantaggiato A, Carinci F, Curioni C. (1996) Apert's syndrome: cephalometric evaluation and considerations on pathogenesis. J. Craniofacial Surg. 7: 23-31.

2. Carinci F, Avantaggiato A, Curioni C. (1994) Crouzon syndrome: cephalometric analysis and evaluation of pathogenesis. Cleft Palate Craniofac. J. 31: 201-209.

3. Bodo M, Carinci F, Baroni T, et al. (1996) Effect of interleukins on Crouzon fibroblast phenotype in vitro. Release of cytokines and IL-6 mRNA expression. Cytokine 8: 772-783.

4. Bodo M, Carinci F, Baroni T, et al. (1997) Apert's syndrome: differential in vitro production of matrix macromolecules and its regulation by interleukins. Eur. J. Clin. Invest. 27: 36-42.

5. Bodo M, Carinci F, Baroni T, et al. (1998) Interleukin pattern of Apert fibroblasts in vitro. Eur. J. Cell Biol. 75: 383-388.

6. Bodo M, Baroni T, Carinci F, et al. (1999) A regulatory role of fibroblast growth factor in the expression of decorin, biglycan, betaglycan and syndecan in osteoblasts from patient with Crouzon's syndrome. Eur. J. Cell Biol. 78: 323-330.

7. Bodo M, Baroni T, Carinci F, et al. (2000) Interleukin secretion, proteoglycans and procollagen-1(I) gene expression in Crouzon fibroblasts treated with basic fibroblast growth factor. Cytokine 12: 280-283.

8. Locci P, Baroni T, Pezzetti F, et al. (1999) Differential in vitro phenotype pattern TGFBl activity and TGFBI mRNA expression in Apert osteoblast. Cell Tissue Res. 279: 475-483.

9. Bodo M, Lilli C, Aisa MC, et al. (2002) Basic fibroblast growth factor: effects on matrix remodeling, receptor expression and transduction pathway in human periosteal fibroblasts with FGFR2 gene mutation. J. Interferon Cytokine Res. 22: 62 1-630.

10. Naski MC, Ornitz DM. (1998) FGF signaling in skeletal development. Front. Biosci. 3: 781-794.

11. Mansukhani A, Bellosta P, Sahni M, Basilico C. (2000) Signaling by fibroblast growth factors (FGF) and fibroblast growth factor receptor 2 (FGFR2)-activating mutations blocks mineralization and induces apoptosis in osteoblasts. J. Cell Biol. 149: 1297-308.

12. Reardon W, Winter RM, Rutland P, Pulleyn LJ, Jones BM, Malcolm S. (1994) Mutations in the fibroblast growth factor receptor 2 gene cause Crouzon syndrome. Nat. Genet. 8: 98-103.
13. Jabs EW, Li X, Scott AF, et al. (1994) Jackson-Weiss and Crouzon syndrome are allelic with mutations in fibroblast growth factor receptor 2. Nat. Genet. 8: 275-279.

14. Wilkie AOM, Slaney SF, Oldridge M, et al. (1995) Apert syndrome results from localized mutations of FGFR2 and is allelic with Crouzon syndrome. Nat. Genet. 9: 165-172.

15. Wilkie AO, Morris-Kay GM. (2001) Genetics of craniofacial development and malformation. Nat. Rev. Genet. 2: 458-468 (review).

16. Lajeunie E, Catala M, Renier D. (1999) Craniosynostosis: from a clinical description to an understanding of bone formation of the skull. Childs Nerv. Syst. 15: 676-680.

17. Francioso F, Carinci F, Tosi L, et al. (2002) Identification of differentially expressed genes in human salivary gland tumors by DNA microarrays. Molecular Cancer Therapeutics. 1: 533-538.

18. Storey JD, Tibshirani R. (2001) Estimating the positive false discovery rate under dependence, with applications to DNA microarrays. Technical report number 2001-28, Stanford, CA: Department of Statistics, Stanford University.

19. Webster MK, Donoghue DJ. (1997) FGFR activation in skeletal disorders: too much of a good thing. Trends Genet. 13: 178-182.

20. Gorry MC, Preston RA, White GJ, et al. (1995) Crouzon syndrome: mutations in two spliceoforms of FGFR2 and a common point mutation shared with Jackson-Weiss syndrome. Hum. Mol. Genet. 4: 1387-1390.

21. De Risi J, Penland L, Brown P, et al. (1996) Use of a cDNA microarray to analyse gene expression patterns in human cancer. Nat. Genet. 14: 457-460.

22. Dell'Era P, Coco L, Ronca R, Sennino B, Presta M. (2002) Gene expression profile in fibroblast growth factor 2-transformed endothelial cells. Oncogene 21: 2433-2440.

23. Bittner M, Meltzer P, Chen Y, et al. (2000) Molecular classification of cutaneous malignant melanoma by gene expression profiling. Nature 406: 536-540.

24. Kahn J, Simon R, Bittner M, et al. (1998) Gene expression profiling of alveolar rhabdomyosarcoma with cDNA microarrays. Cancer Res. 58: 5009-5013.

25. Schaub R, Dupont B, Roodman GD, Leach RJ, Reddy SV. (2000) Assignment of OSTF1 to human chromosome bands $12 \mathrm{q} 24.1->\mathrm{q} 24.2$ by in situ hybridization. Cytogenet. Cell. Genet. 88: 87-88.

26. McPherron AC, Lawler AM, Lee SJ. (1999) Regulation of anterior/posterior patterning of the axial skeleton by growth/ differentiation factor 11. Nat. Genet. 22: 260-264.

27. Volinia S, Patracchini P, Otsu M, et al. (1992) Chromosomal localization of human p85-alpha, a subunit of phosphatidylinositol 3-kinase, and its homologue p85-beta. Oncogene 7: 789-793.

28. Berchtold MW, Egli R, Rhyner JA, Hameister H, Strehler EE. (1993) Localization of the human bona fide calmodulin genes CALM1, CALM2, and CALM3 to chromosomes 14q24- q31, 2p21.1-p21.3, and 19q13.2-q13.3. Genomics 16: 461-465.

29. Morris CM, Bodger MP. (1993) Localization of the human poly(A)-binding protein gene (PABl) to chromosomal regions 3q22-q25, 12q13-q14, and 13q12-q13 by in situ hybridization. Genomics 15: 209-211.

30. Claret FX, Hibi M, Dhut S, Toda T, Karin M. (1996) A new group of conserved coactivators that increase the specificity of AP-1 transcription factors. Nature 383: 453-457.

31. Liu M, Xie Z, Price DH. (1998) A human RNA polymerase II transcription termination factor is a SWI2/SNF2 family member. J. Biol. Chem. 273: 25541-25544.

32. Ogawa K, Tashima M, Yumoto Y, et al. (1990) Coding sequence of human placenta cofilin cDNA. Nucleic Acids Res. 18: 7169.

33. Goldschmidt-Clermont PJ, Janmey PA. (1991) Profilin, a weak CAP for actin and RAS. Cell 66: 419-421.

34. Carinci P, Becchetti E, Bodo M. (2000) Role of the extracellular matrix and growth factors in skull morphogenesis and in the pathogenesis of craniosynostosis. Int. J. Dev. Biol. 44: 715-723.

35. Arena N, Venti G, Becchetti E, et al. (1991) Characterization of the cytoskeleton in human normal and otosclerotic osteoblast-like cells. Acta Anat. 141: 311-315. 